\title{
Non Aggregated Colloidal Silver Nanoparticles for Surface Enhanced Resonance Raman Spectroscopy
}

\author{
Aoife Power \\ Technological University Dublin, aoife.power@tudublin.ie \\ John Cassidy \\ Technological University Dublin, john.cassidy@tudublin.ie \\ Tony Betts \\ Technological University Dublin, anthony.betts@tudublin.ie
}

Follow this and additional works at: https://arrow.tudublin.ie/scschcpsart

Part of the Analytical Chemistry Commons

\section{Recommended Citation}

Power, A., Cassidy, J. \& Betts, T. (2011) Non Aggregated Colloidal Silver Nanoparticles for Surface Enhanced Resonance Raman Spectroscopy. The Analyst, vol. 136,2794-2801. doi:10.1039/c1an15250e

This Article is brought to you for free and open access by the School of Chemical and Pharmaceutical Sciences at ARROW@TU Dublin. It has been accepted for inclusion in Articles by an authorized administrator of ARROW@TU Dublin. For more information, please contact arrow.admin@tudublin.ie, aisling.coyne@tudublin.ie, gerard.connolly@tudublin.ie.

Funder: ABBEST PhD Scholarship Programme of the Technological University Dublin

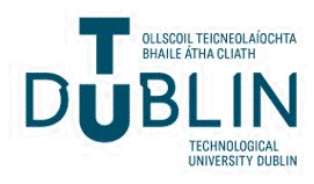


Non Aggregated Colloidal Silver Nanoparticles for Surface Enhanced Resonance Raman Spectroscopy.

Aoife C. Power ${ }^{\text {a., c. }}$, Anthony J. Betts ${ }^{\text {b., c. }}$ and John F. Cassidy ${ }^{\text {a., c. }}$

${ }^{a}$ School of Chemical and Pharmaceutical Sciences, Dublin Institute of Technology, Kevin St., Dublin 8, Ireland.

${ }^{\mathrm{b}}$ Directorate of Research \& Enterprise, Dublin Institute of Technology, 143-149 Rathmines Rd., Dublin 6, Ireland.

c Applied Electrochemistry Group, Focas Institute, Dublin Institute of Technology, Camden Row Dublin 8, Ireland.

Silver nanoparticles with a tuneable $\lambda$ max were produced as colloids by heterogeneous nucleation. The synthesis process is both fast and repeatable, producing stable PVA capped nanoparticles. The colloid's effectiveness in the SERRS system was investigated using Rhodamine 6G, R6G, Crystal Violet, CV, and Malachite Green, MG, as probe molecules. A clear sensing trend was observed, where the Raman signal emitted was significantly enhanced by the addition of silver nanoparticles. A build up of signal intensity is observed until an optimum ratio is achieved, followed by a decline in signal intensity as the concentration of nanoparticles is further increased. The sensing trend appeared to be dependant on the structure of these model molecules with similarly structured compounds exhibiting similar trends. Thus a maximum enhancement with the Ag : analyte molar ratio of $\sim$ 5.56 : 1, was seen for $\mathrm{CV}$ and MG whereas R6G had a maximum enhancement at the $\mathrm{Ag}:$ analyte molar ratio of $\sim 2.25: 1$.

\section{Introduction}

Nanotechnology's role in the development of sensors has been mounting rapidly; this is especially the case for metallic nanostructures as they often exhibit novel physiochemical properties that differ greatly from their 'bulk' counterparts. The role of silver nanoparticles in the development of sensors in terms of both sensitivity and selectivity is the subject of much research. Metal nanoparticles, and in particular silver nanoparticles, have proved to be good candidates for use in Surface Enhanced 
Resonance Raman Scattering (SERRS), exhibiting the dual roles of both substrate and signal enhancers ${ }^{\mathbf{1 - 2}}$.

Raman spectroscopy is both a non-destructive and non-invasive vibrational spectroscopic technique, which utilises the inelastic scattering of the monochromatic light achieved by laser excitation. Raman elucidates the molecular profiles of systems by measuring frequency shifts that adhere to a selection rule, whereby changes in polarisability of the analyte is required ${ }^{3-6}$. However, Raman's usefulness in practical applications is hindered by the very weak signal produced by Raman scattering as typically only one in $10^{6}-10^{8}$ photons undergo inelastic (Raman) scattering when interacting with a sample ${ }^{7}$. This results in the need to use high power lasers or long sampling times to counteract the inefficient scatter for adequate spectral acquisition that makes the technique unsuitable in real world applications 8 .

Resonance Raman discovered by Shorygin, had a significant impact on Raman's usefulness, as an increase in the intensity of the measured Raman signal was observed when the frequency of the excitation radiation overlapped with the absorption band of an analyte, improving the techniques detection range. ${ }^{9}$

Surface Enhanced Raman Scattering (SERS) is a technique used to amplify inherently weak Raman signal and involves a catalyst, typically of the nanoscale. Nanoparticles of noble metals have been found particularly useful as they exhibit surface plasmon resonance (SPR) ${ }^{10-11}$. This SPR involves a collective oscillation of the conduction electrons in resonance with certain frequencies of incident light, where the plasmon resonance of the metallic substrate (colloids) provides the intense optical frequency fields responsible for the electromagnetic contribution to SERS method ${ }^{\mathbf{1 2}-16}$. Interest in the application of SERS as an effective analytical tool is increasing, with the potential of the development of highly selective and sensitive detection ${ }^{17-18}$. Often it is noted in the literature that prior to sampling, metallic colloids undergo the addition of an aggregating agent, which results in destabilising the colloid and thus the creation of inter-particle junctions or hotspots to improve the Raman scatter ${ }^{\mathbf{1 3},{ }^{19-20}}$. It follows that by coupling these two means of signal enhancement, resonance Raman and SERS, the sensitivity of Raman as an analytical is further enhanced. However, these destabilised Nanoparticles aggregate in a non uniform manner and are prone to coagulate and precipitate, which increases the difficulty of use and ultimately results in SERS signals that are difficult to reproduce ${ }^{10,12,17-18,21-23}$. 
As a result, this work examined the use of non aggregated silver Nanoparticles in colloids of tuneable $\lambda$ max. These colloids were prepared by heterogeneous nucleation 24-25. First a seed solution was prepared by the chemical reduction of silver nitrate $\left(\mathrm{AgNO}_{3}\right)$ with sodium borohydride $\left(\mathrm{NaBH}_{4}\right)$ in the presence of a stabiliser and capping agent, polyvinyl alcohol (PVA) ${ }^{26}$. The seed solution was then added to a mixture of tri sodium citrate (TSC) and hydrazine $\left(\mathrm{H}_{4} \mathrm{~N}_{2}\right)$, (two weak reducing agents), and agitated to produce a homogeneous 'growth' solution. To this, set concentrations of $\mathrm{AgNO}_{3}$ were added, producing different colloids by crystal growth. The different concentrations of excess $\mathrm{AgNO}_{3}$ that are added to the growth mixture determine the size and the morphological characteristics, of the nanoparticles in the dispersion.

\section{Experimental}

Silver nitrate (purum p.a. > 99.0\%), sodium borohydride (reagent Plus 99\%) polyvinyl alcohol (PVA), (99 +\% hydrolyzed, typical M.W. 89000-98000 gmol ${ }^{-1}$ ), tri sodium citrate (TSC), (purum p.a., $\geq 99.0 \%$ ), hydrazine, (reagent grade, $\mathrm{N}_{2} \mathrm{H}_{4} 50$ $60 \%$ ), crystal violet, (ACS reagent, $\geq 90.0 \%$ anhydrous basis), malachite green, (indicator Riedel-de Haën), Rhodamine 6G, (Dye content $95 \%$ ), were all purchased from Sigma Aldrich, and used as received without further purification.

The experimental process in this study comprised of a number of distinct steps. First the preparation and characterisation of the colloids was performed, followed by their application as SERS substrates.

Characterisation of the silver nanoparticles involved several techniques including UVVis and dynamic light scattering (DLS), which were conducted using a Perkin Elmer, Lambda 900 Spectrometer, and a Malvern nano series Zetasizer respectively. Transmission Electron Microscope (TEM or STEM) images were captured with a JEOL, 100CX Transmission Electron Microscope or a Hitachi SU 6600 FESEM.

As the maximum SERS enhancement is expected to take place when the $\lambda$ max of the localised SPR of the nanoparticles is close to that of the excitation (laser) wavelength, both the Raman scattered photon and the incident photon are strongly enhanced ${ }^{\mathbf{3 - 4}, 16}$. The blue colloid $(\lambda \max , 670 \mathrm{~nm})$ was used to enhance measurements excited by the $660 \mathrm{~nm}$ laser. 
SERS active measurements were conducted on three model analytes, Crystal Violet (CV), Malachite Green (MG) and Rhodamine 6G (R6G), and were performed with a Horiba Jobin Yvon LabRAM HR 800. SERRS excitation was provided by Solid State Diode laser with a maximum power of $100 \mathrm{~mW}$. The spectrograph was equipped with a 600 grooves $/ \mathrm{mm}$ grating, and all measurements were performed with a $100 \mu \mathrm{m}$ entrance slit with the laser at $1 \%$ strength and using the $\mathrm{x} 10$ objective lense. The spectral region of investigation was dependent on the analyte, $700-1200 \mathrm{~cm}^{-1}$ for $\mathrm{CV}$, $1000-1800 \mathrm{~cm}^{-1}$ for MG and $200-2000 \mathrm{~cm}^{-1}$ for R6G. Emphasis was placed on the use of the technique as an analytical tool, and as short sampling times are the ideal, in each case the exposure time was 5 seconds with the accumulation set to 30 , ensuring that the total sampling time was never greater than 5 minutes.

\section{Results and Discussion}

\section{Preparation of colloids}

The synthesis methods proposed below are completely reliable, providing the experiment is conducted with proper care (i.e. slow drop-wise addition of reagents and the use of properly cleaned glass ware). The prepared reagents, with the exception of sodium borohydride, will last several days to months if stored appropriately (e.g. photosensitive silver nitrate solutions should be stored in opaque vessels). As sodium borohydride decomposes in water (equation 1); it is necessary that the sodium borohydride solution should be freshly prepared. It should be noted that the best results are achieved when all reagent solutions are freshly made.

$$
\mathrm{NaBH}_{4}(\mathrm{aq})+4 \mathrm{H}_{2} \mathrm{O}(\mathrm{l}) \rightarrow \mathrm{Na}\left[\mathrm{B}(\mathrm{OH})_{4}\right](\mathrm{aq})+4 \mathrm{H}_{2}(\mathrm{~g})
$$

The silver colloids were produced using heterogeneous nucleation. Here the seed solution was once more prepared by the chemical reduction of $\mathrm{AgNO}_{3}$ with $\mathrm{NaBH}_{4}$ in the presence of polyvinyl alcohol (PVA) ${ }^{\mathbf{2 6}}$ a stabiliser and capping agent. It should be noted that this seed synthesis requires great care with the addition of the reducing agent, in order to produce a suitable seed and hence coloured colloids. 


\section{Seed production}

$2 \mathrm{~cm}^{3} 0.001 \mathrm{M} \mathrm{AgNO}_{3}$ was added to $2 \mathrm{~cm}^{3}$ of PVA (1\% w/v) and mixed well. To this while ensuring that the mixture underwent constant agitation $2 \mathrm{~cm}^{3}$ of $0.001 \mathrm{M} \mathrm{NaBH}_{4}$ was added drop wise. Best results were achieved when the resulting seed solution was a golden yellow.

\section{Fabrication of coloured colloids}

$1 \mathrm{~cm}^{3}$ of the seed solution, $3 \mathrm{~cm}^{3} 0.1 \mathrm{M}$ TSC and $5 \mathrm{~cm}^{3} 0.1 \mathrm{M} \mathrm{H} \mathrm{H}_{4} \mathrm{~N}_{2}$ were added to 1 $\mathrm{cm}^{3} 1 \%$ PVA (again ensure this mixture undergoes constant agitation). To produce the coloured colloids, set volumes of $0.001 \mathrm{M} \mathrm{AgNO}_{3}$ should be added to this 'growth mixture' as outlined in table 1 . The hydrazine acts as a mild reducing agent with the citrate also acting to both reduce and stabilise the colloid with the PVA.

\section{Colloid characterisation}

\section{UV-Vis analysis}

The absorption spectrum of the 'seed' colloid, shown in Figure 1; this indicates the production of nanoparticles, as a plasmon absorption band at $\sim 400 \mathrm{~nm}$ is typical of silver nanoparticles ${ }^{26}$.

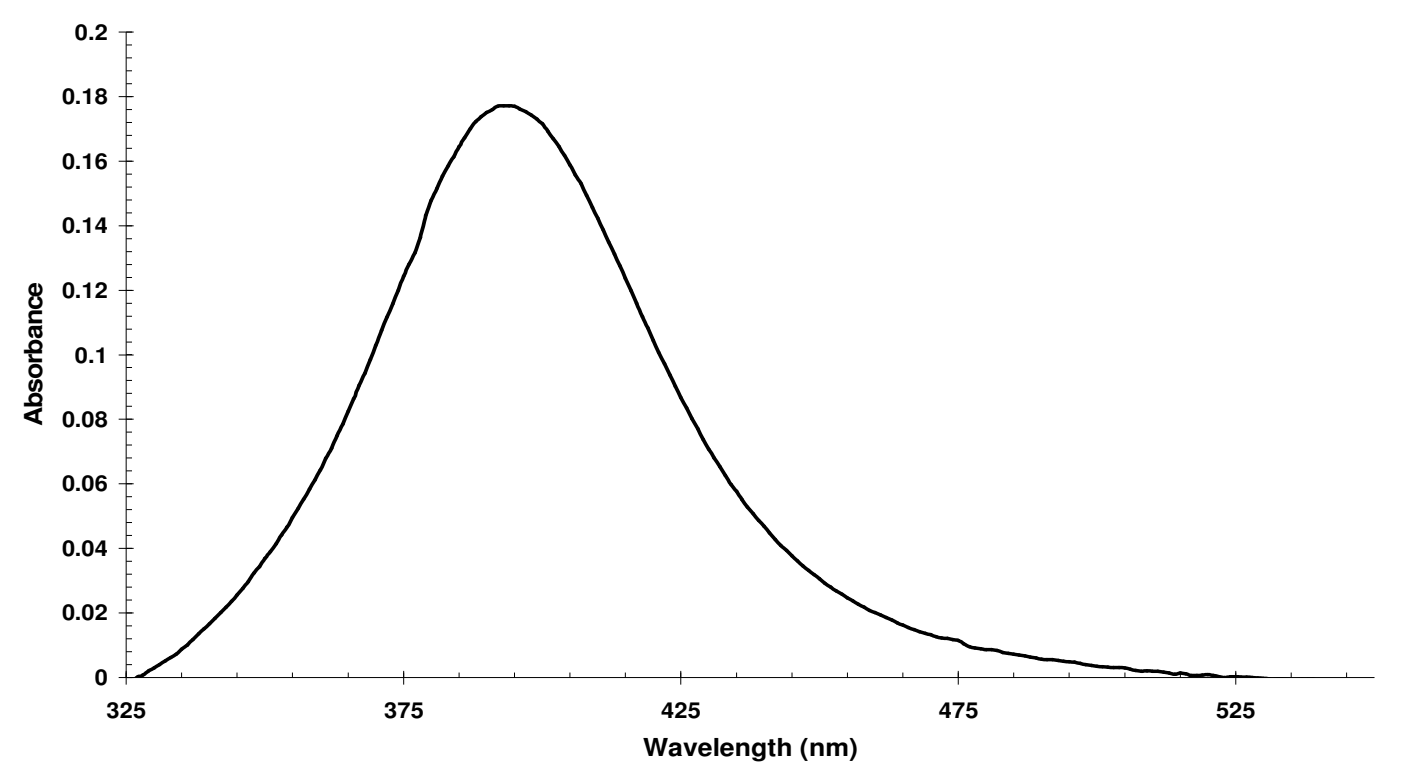

Figure 1. UV-Vis spectrum of stable aqueous colloidal Ag Seed solution, with $\lambda$ max of $393 \mathrm{~nm}$. $\left[\mathrm{Ag}^{+}\right]=0.001 \mathrm{M}\left(2.0 \mathrm{~cm}^{3}\right),[\mathrm{PVA}]=1 \% \mathrm{wt} / \mathrm{wt}\left(2.0 \mathrm{~cm}^{3}\right),\left[\mathrm{NaBH}_{4}\right]=0.001 \mathrm{M}\left(2.0 \mathrm{~cm}^{3}\right)$, with a total volume of $6 \mathrm{~cm}^{3}$. 
Such plasmon bands are a result of the unique physical properties of the nanoparticles themselves. When an external electro-magnetic field such as light is applied to a metal, the conduction electrons move collectively so as to screen the perturbed charge distribution in what is known as plasmon oscillation. The Surface Plasmon Resonance (SPR) is therefore a collective excitation mode of the plasmon localized near the metal surface. In the case of a metal nanoparticle, the surface plasmon mode is 'restricted' due to the small dimensions to which the electrons are confined ${ }^{28}$, i.e. the surface plasmon mode must conform to the boundaries of the dimensions of the nanoparticle ${ }^{29}$. Therefore, the resonance frequency of the surface plasmon oscillation of the metal nanoparticle is different from the plasmon frequency of the bulk metal. Surface interactions can alter the optical properties and influence the spectral profile of the light scattered by the SPR of the metal nanoparticles. This feature can be employed as an indicator in sensing interactions. Among the metal nanoparticles known to exhibit SPR, silver nanoparticles have an especially strong SPR. Particle size may be determined using Mie theory, which solves Maxwell's equations ${ }^{\mathbf{3 0}}$, that in turn describes the extinction spectra (extinction $=$ scattering + absorption) of spherical particles of arbitrary size. The spectrum illustrated in Figure 1 displays the characteristic SPR of spherical silver nanoparticles, commonly seen in the literature ${ }^{27}$, 31-32. 


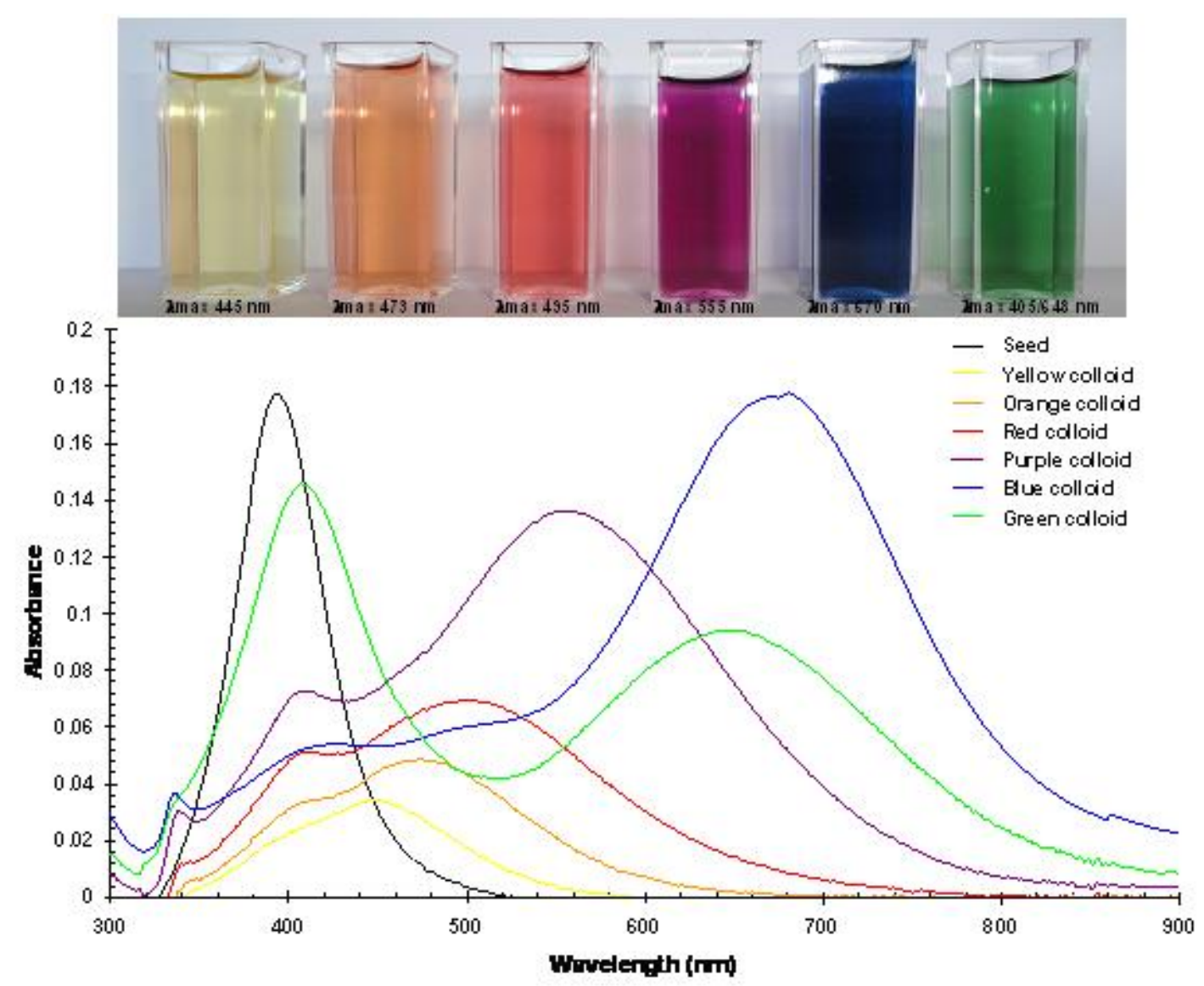

Figure 2. UV-Vis spectra of aqueous Ag colloids. General make up of colloids [PVA] = 1\% wt/wt $(1.0$ $\left.\mathrm{cm}^{3}\right)$, seed solution $\left(1.0 \mathrm{~cm}^{3}\right),[\mathrm{TSC}]=0.1 \mathrm{M}\left(3.0 \mathrm{~cm}^{3}\right),\left[\mathrm{H}_{4} \mathrm{~N}_{2}\right]=0.001 \mathrm{M}\left(5.0 \mathrm{~cm}^{3}\right)+\left[\mathrm{AgNO}_{3}\right]=$ $0.001 \mathrm{M}\left(\mathrm{X} \mathrm{cm}^{3}\right)$, with a total volume of $10+\mathrm{X} \mathrm{cm}^{3}$.

Figure 2 shows the spectra of both the seed colloid and the colloids of larger particles 'grown' from the seed. It should be noted that for smaller particle size ranges e.g. Yellow, a narrower/sharper absorbance band is observed, while Blue with a wider particle size range has clearly broader peaks. The $\lambda$ max shifts position with changes in the nanoparticles size and shape (Figure 2).

The different natures of each colloid is also highlighted in the UV-Vis spectra of the green colloid where two distinct absorbance peaks are observed as a result of the interaction of two species (type) of nanoparticles, i.e. the larger blue nanoparticles and the smaller yellow nanoparticles. These are the most likely species contributing to the green as the peaks are seen to have similar $\lambda$ max's - Yellow $445 \mathrm{~nm}$, Blue 670nm, Green band 1, $405 \mathrm{~nm}$, band 2, $648 \mathrm{~nm}$., while the other colloids exhibit just one distinct band (which can however display a shoulder, but this could be attributed to band broadening due to the wider particle size range). 


\section{Dynamic light scattering, DLS}

Size analysis by DLS utilises the Brownian motion that particles, emulsions, and molecules in suspension undergo as a result of bombardment by solvent molecules. If the particles are illuminated with a laser, the intensity of the scattered light fluctuates at a rate that is dependent upon the size of the particles. This is because smaller particles are "hit" more frequently by the solvent molecules and move more rapidly. Analysis of these intensity fluctuations yields the velocity of the Brownian motion and hence the particle size using the Stokes-Einstein relationship ${ }^{33}$.

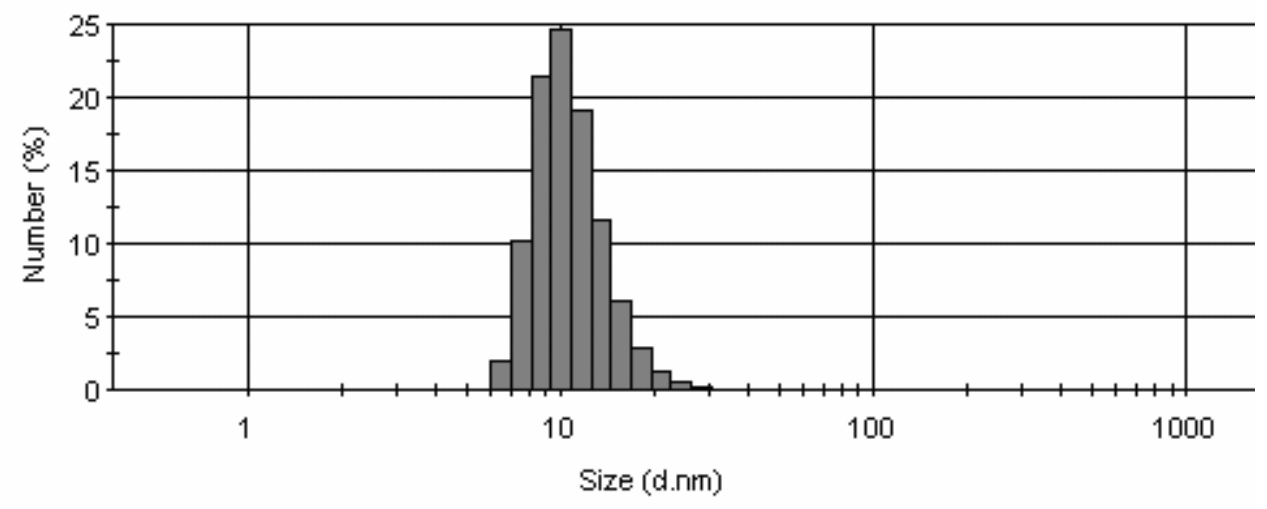

Figure 3 a. Distribution of particle diameters within the Yellow Ag colloid, $\left(x=0.4 \mathrm{~cm}^{3}\right.$ see table 1).

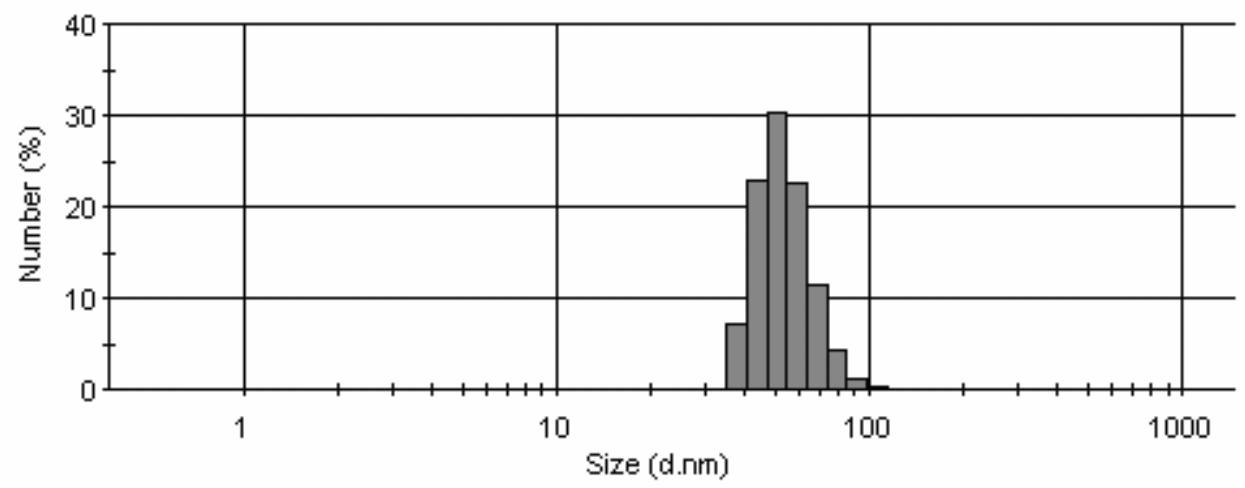

Figure $3 \mathrm{~b}$. Distribution of particle diameters within the Blue Ag colloid, $\left(\mathrm{x}=6.0 \mathrm{~cm}^{3}\right.$ see table 1).

Colour

Yellow

Orange

Red

Purple

Blue

Green
UV-Vis $\lambda$ max

$\mathrm{AgNO}_{3} \operatorname{added}\left(\mathrm{X} \mathrm{cm}^{3}\right)$

$$
0.4
$$

0.8

1.3

2.5

6.0

21.5
$445 \mathrm{~nm}$

$473 \mathrm{~nm}$

$495 \mathrm{~nm}$

$555 \mathrm{~nm}$

$670 \mathrm{~nm}$

$405 \mathrm{~nm} \& 648 \mathrm{~nm}$
DLS

DLS

\section{Particle Size Range}

$$
\begin{gathered}
6-28 \mathrm{~nm} \\
11-38 \mathrm{~nm}
\end{gathered}
$$

$11 \mathrm{~nm}-60 \mathrm{~nm}$

$15 \mathrm{~nm}-50 \mathrm{~nm}$

$37 \mathrm{~nm}-105 \mathrm{~nm}$

$11-250 \mathrm{~nm}$

\section{Average Size Range}

$10-13 \mathrm{~nm}$

$19-20 \mathrm{~nm}$

20-23 nm

31-33 nm

$58-60 \mathrm{~nm}$
$91.1 \% 20-21 \mathrm{~nm} \& 8.9 \% \geq 60 \mathrm{~nm}$ 
Table 1. Summary of Colloids UV-Vis $\lambda$ max's and DLS results.

A breakdown of the UV-Vis, DLS results is supplied in table 1 with graphical illustrations highlighting the DLS results of both the yellow and blue colloids in figures $3 \mathrm{a}$ and $3 \mathrm{~b}$ respectively. It can be seen that the $\lambda \max$ shifts with particle size as seen elsewhere in the literature ${ }^{10-11,24-25,30-32}$.

\section{Electron microscopy}

An image of the nanoparticles, (Figure 4) was obtained from the interaction of the coating and the beam of electrons transmitted through the coating. Before analysis, the colloidal samples (prepared in the same manner as outlined in Figure 2 and Table 1) were diluted in ethanol and sonicated for 30 mins, before being cast onto the TEM grid (Agar scientific, holey carbon 200 mesh $(\mathrm{Cu})$ ) by drop coating. The average diameters of the nanoparticles were determined, using Zeiss axiovision software ${ }^{\mathbf{3 7}}$ and correlated well with the DLS results.

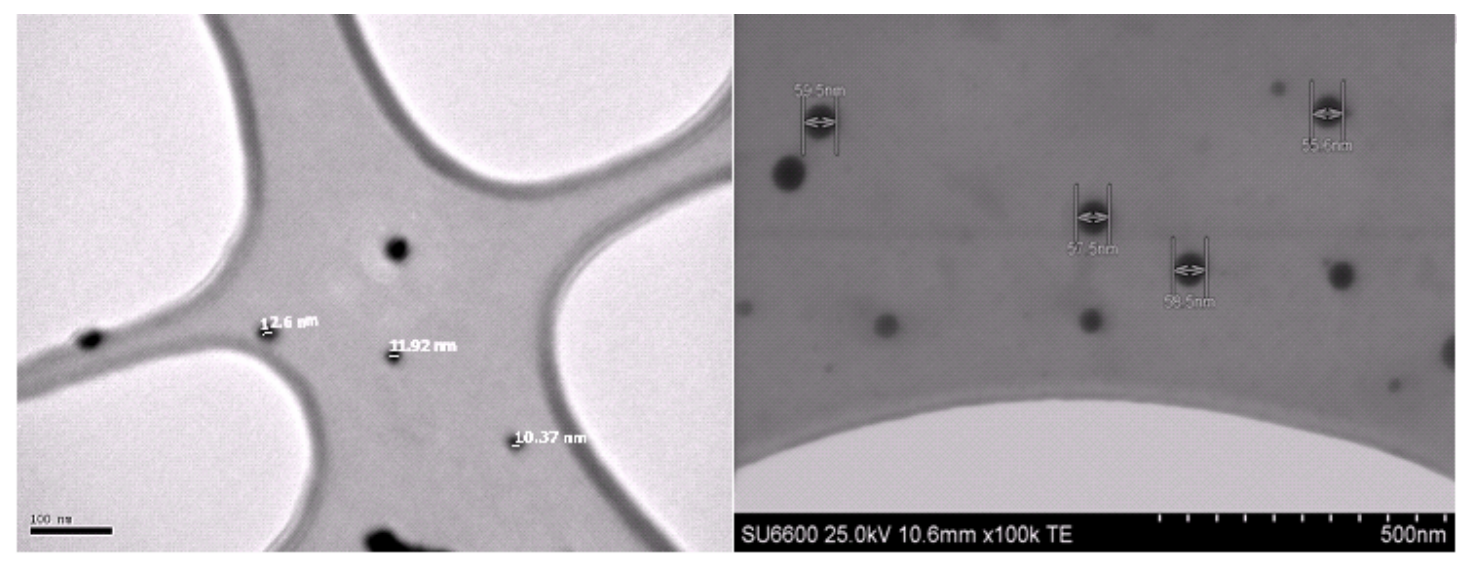

Figure 4. On the left a TEM image of silver nanoparticles (yellow colloid) with an average diameter range of $10-13 \mathrm{~nm}$, and on the right a STEM image of silver nanoparticles (blue colloid) with an average diameter range of 58-60 nm.

\section{Colloid Stability}

The prepared colloids proved highly stable when stored appropriately (best results observed when stored in dark), with repeat spectroscopic (UV-Vis) analysis of the individual colloids overtime (after a 6 month period) correlating well with initial analysis. 


\section{Dye and colloid interaction}

It is necessary to determine the effect of the analyte (if any) on the colloids as unwanted aggregation of nanoparticles despite the use of PVA as a stabiliser ${ }^{\text {33-36 }}$ remains a concern. DLS was used to monitor the particles with increasing concentrations of dye.

(a)

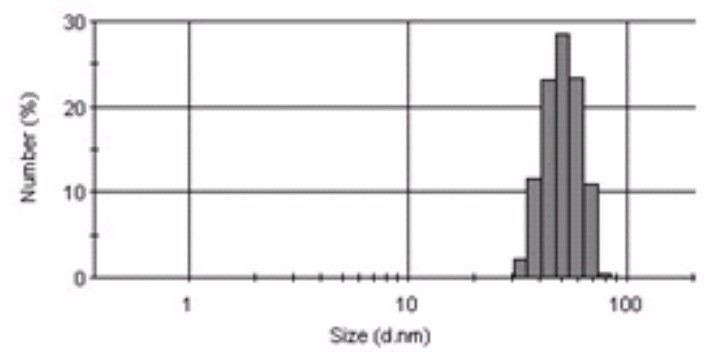

(b)

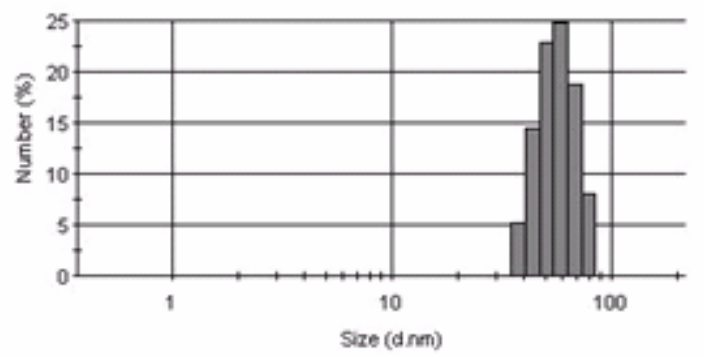

Figure 5. Effect of the Dye on the particle size range of the colloidal nanoparticles, (a) silver colloid (blue) $[\mathrm{Ag}]=1 \times 10^{-5} \mathrm{M}$, (b) silver colloid (blue) $[\mathrm{Ag}]=1 \times 10^{-5} \mathrm{M}$ with excess crystal violet $[\mathrm{CV}]=5 \mathrm{x}$ $10^{-5} \mathrm{M}$.

Some aggregation occurs when the analyte is present; however the extent of aggregation is limited with the bulk of the nanoparticles maintaining an average diameter range of 58-60 $\mathrm{nm}$.

\section{SERRS measurements}

The performances of the colloids as SERRS substrates were evaluated by their SERRS activity using Crystal Violet (CV), Malachite Green (MG), and Rhodamine 6G (R6G) as model compounds (Figure 6). All dyes were made up to $10^{-4} \mathrm{M}$ aqueous solutions (further dilution occurred during the sampling process, meaning the sample's actual concentration was $3.33 \times 10^{-5} \mathrm{M}$.) as water molecules produce very weak Raman scatter, which allows analytes in aqueous solutions to be readily detected at low concentrations. The liquid samples and substrates also enabled reproducible sampling where the constant disorder/movement (Brownian motion) of both the analyte and substrate produced a stable and reproducible system. Note the overall concentration of the analyte for the study was maintained through out the experimental process by preparing separate samples that for each measurement ensuring that the total volume of the samples remained consistent. This, coupled with the use of the confocal microscope on the Horiba Jobin Yvon LabRAM HR 800, has the advantage of creating a high power density in a small volume with a relatively weak laser, where the particles readily move in and out of the analysis volume during 
the measurement. This reduces problems with photodegradation that can be seen with other SERS substrates ${ }^{17}$.

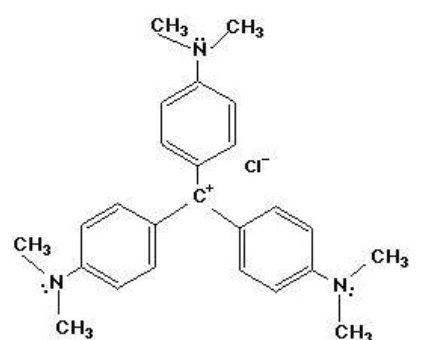

Crystal Violet

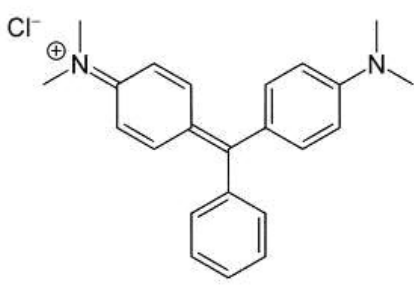

Mal achite Green<smiles></smiles>

Rhodamine $6 \mathrm{G}$

Figure 6. Molecular structures of Crystal Violet, Malachite Green and Rhodamine 6G.

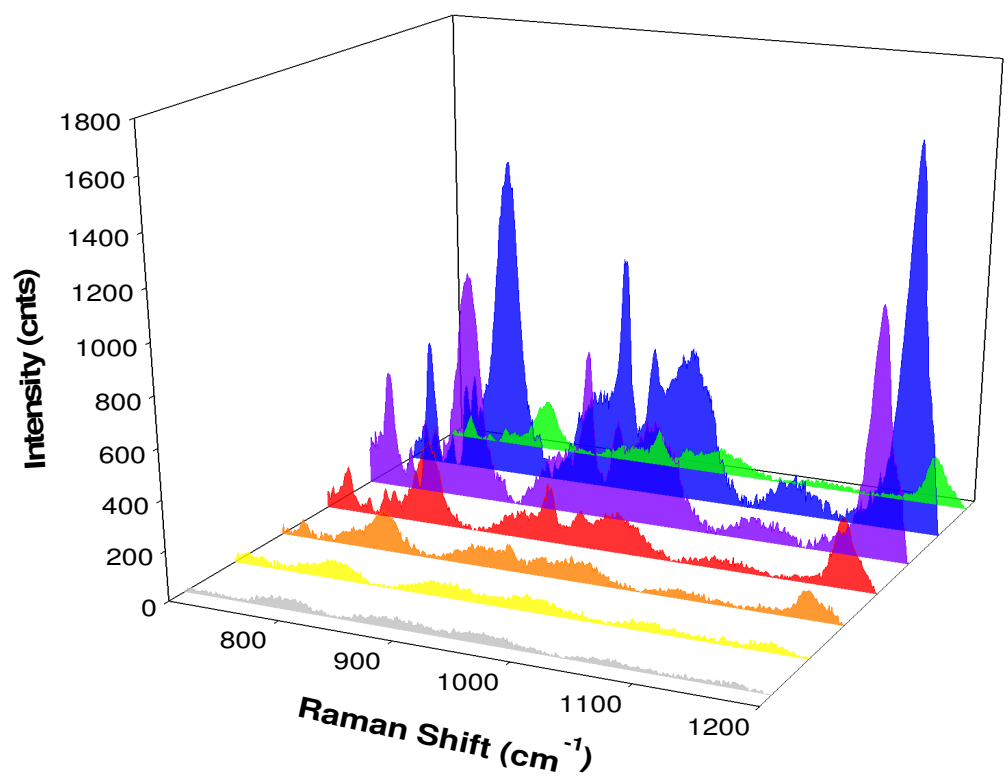

CV only
CV + Yellow Nps
CV + Orange NPs

CV + Red NPs

CV + Purple NPs

CV + Blue NPs

CV + Green NPs

Figure 7. Raman Spectra of $3.33 \times 10^{-5} \mathrm{M}$ CV mixed with each colloid

with excitation by the $660 \mathrm{~nm}$ laser. $[\mathrm{Ag}]=11 \times 10^{-5} \mathrm{M}$, for colours makeup please refer to Table 1 .

Figure 7 shows the SERRS spectra of CV with deliberately added silver nanoparticles

prepared as stated in table 1 , where the $\mathrm{CV}$ concentration was $3.33 \times 10^{-5} \mathrm{M}$ and the silver concentration was $11 \times 10^{-5} \mathrm{M}$ in each case, as expected. Based on the literature 3-4, 16, initial analysis of CV with the $660 \mathrm{~nm}$ laser shown in figure 7 indicated that the blue colloid was the most effective substrate because it's nanoparticles LSPR (localised SPR) was slightly longer than that of the excitation wavelength. Similarly the purple colloid was found to be the most effective (Figure 8) with excitation by the $532 \mathrm{~nm}$ laser for enhancing the signal. 


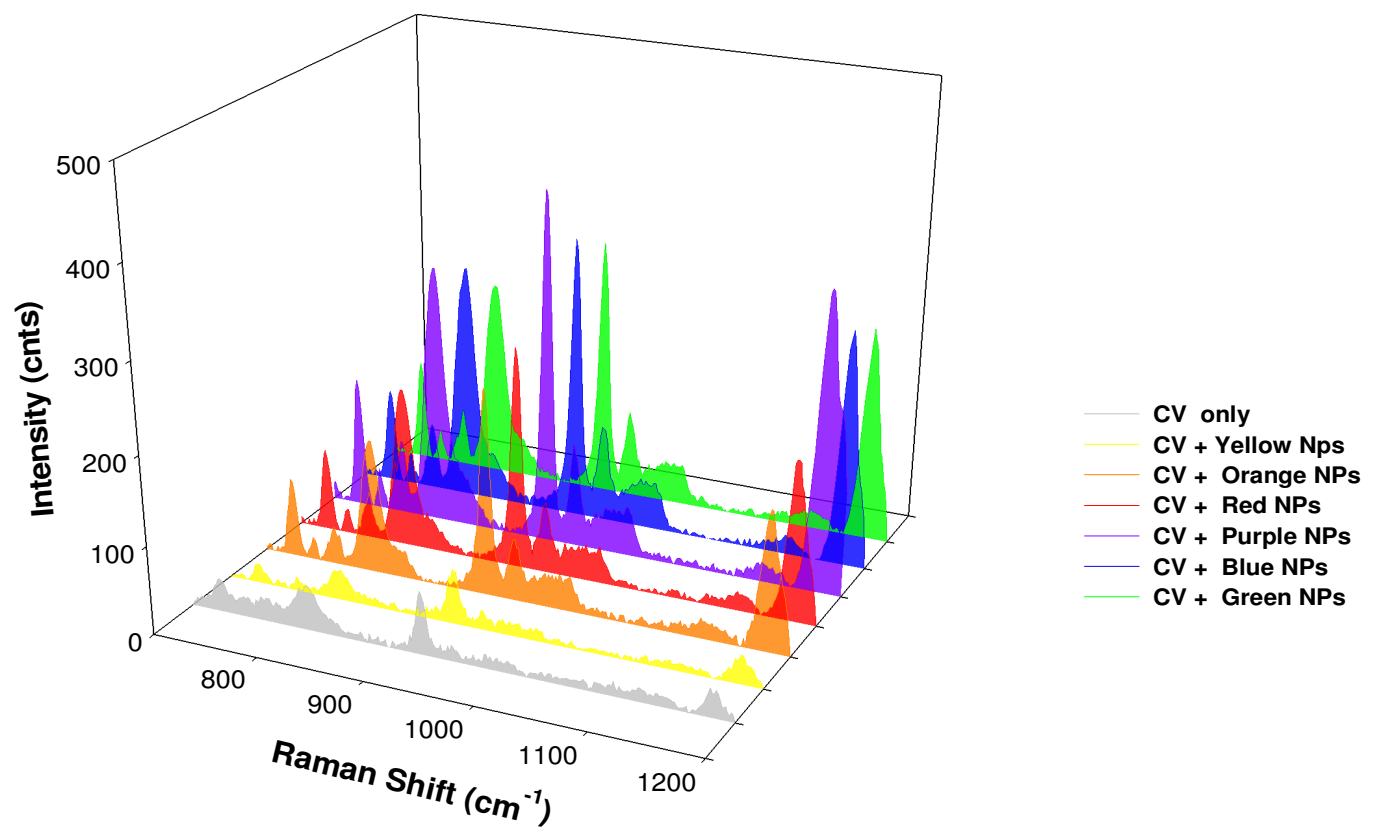

Figure 8. Raman Spectra of $3.33 \times 10^{-5} \mathrm{M}$ CV mixed with each colloid with excitation by the $532 \mathrm{~nm}$ laser. $[\mathrm{Ag}]=11 \times 10^{-5} \mathrm{M}$, for colours makeup please refer to Table 1 .

Similar results were observed for Malachite Green and Rhodamine 6G, (note R6G measurements were only conducted at the $660 \mathrm{~nm}$ excitation wavelength as the dye fluoresces when excited at $532 \mathrm{~nm}$.) Therefore, for all measurements at the $660 \mathrm{~nm}$ excitation, the blue colloidal nanoparticles were used as the SERRS substrate while the purple colloidal nanoparticles were used for measurements excited by the $532 \mathrm{~nm}$ laser.

\section{Sensing Trend}

The relevant colloids were then added incrementally to a set concentration of the model compounds, and their Raman spectra recorded with each increase of the blue colloidal nanoparticles. In doing so, the following trends were observed (figures 813). Figure 9 shows the SERRS spectra of $3.33 \times 10^{-5} \mathrm{M} \mathrm{CV}$ with the blue colloidal nanoparticles prepared as described in table 1. Figure 10 shows the peak emissions observed with increasing concentrations of silver at certain wavelengths taken from figure 9. Similarly figure 12 comes from figure 11 where the SERRS spectra of $3.33 \mathrm{x}$ $10^{-5} \mathrm{M}$ MG with the blue colloidal nanoparticles is displayed and figure 14 comes 
from figure 13 where the SERRS spectra of $3.33 \times 10^{-5} \mathrm{M}$ R6G with the blue colloidal nanoparticles is illustrated.

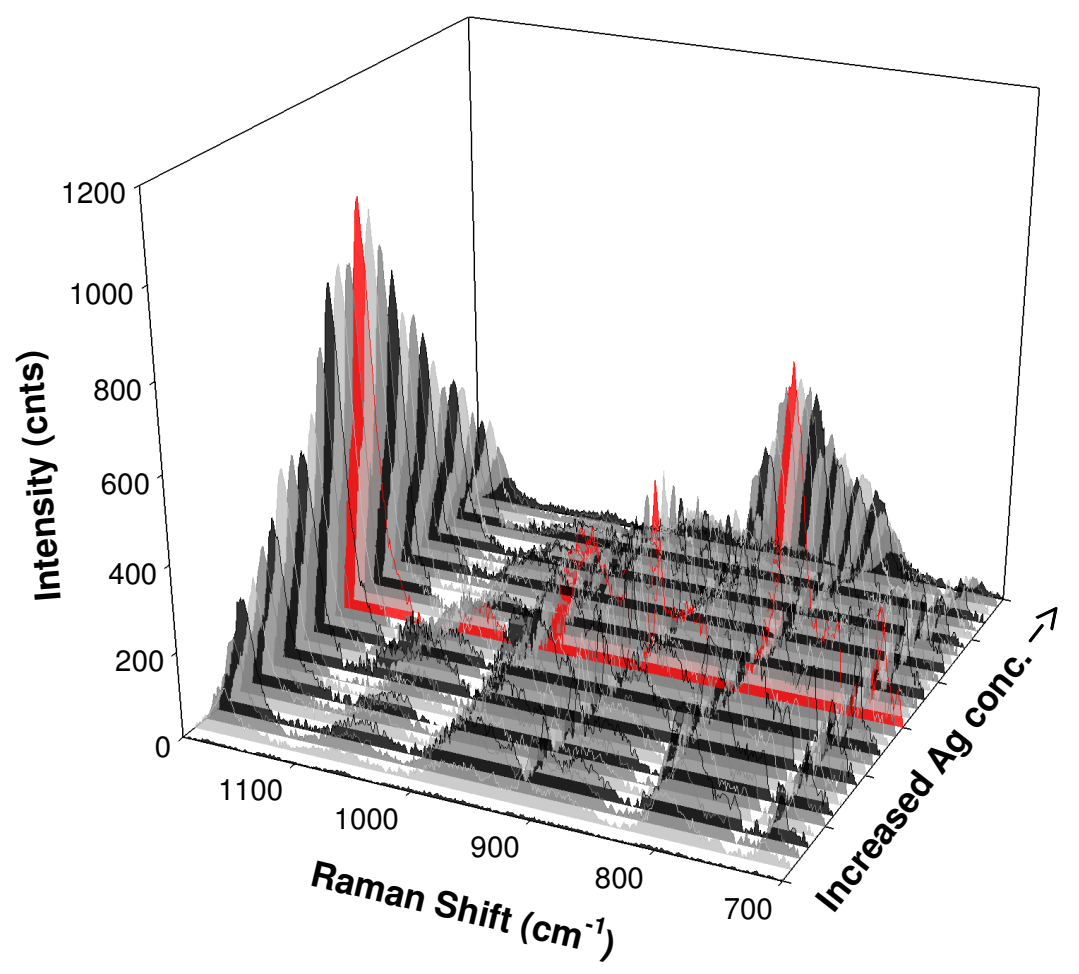

Figure 10. Raman Spectra of $3.33 \times 10^{-5} \mathrm{MCV}$ with increasing concentrations of the blue colloidal nanoparticles with excitation by the $660 \mathrm{~nm}$ laser. The bands of the Raman spectra may be assigned as follows; $\sim 722 \mathrm{~cm}^{-1}$, In plane benzene ring bend, stretch; $\sim 800 \mathrm{~cm}^{-1}$, out of plane aromatic C-H 'breathing'; $\sim 914 \mathrm{~cm}^{-1}$, ring skeletal radial vibration; $\sim 1170 \mathrm{~cm}^{-1}$, in plane aromatic C-H bending vibration $^{39-40}$. 


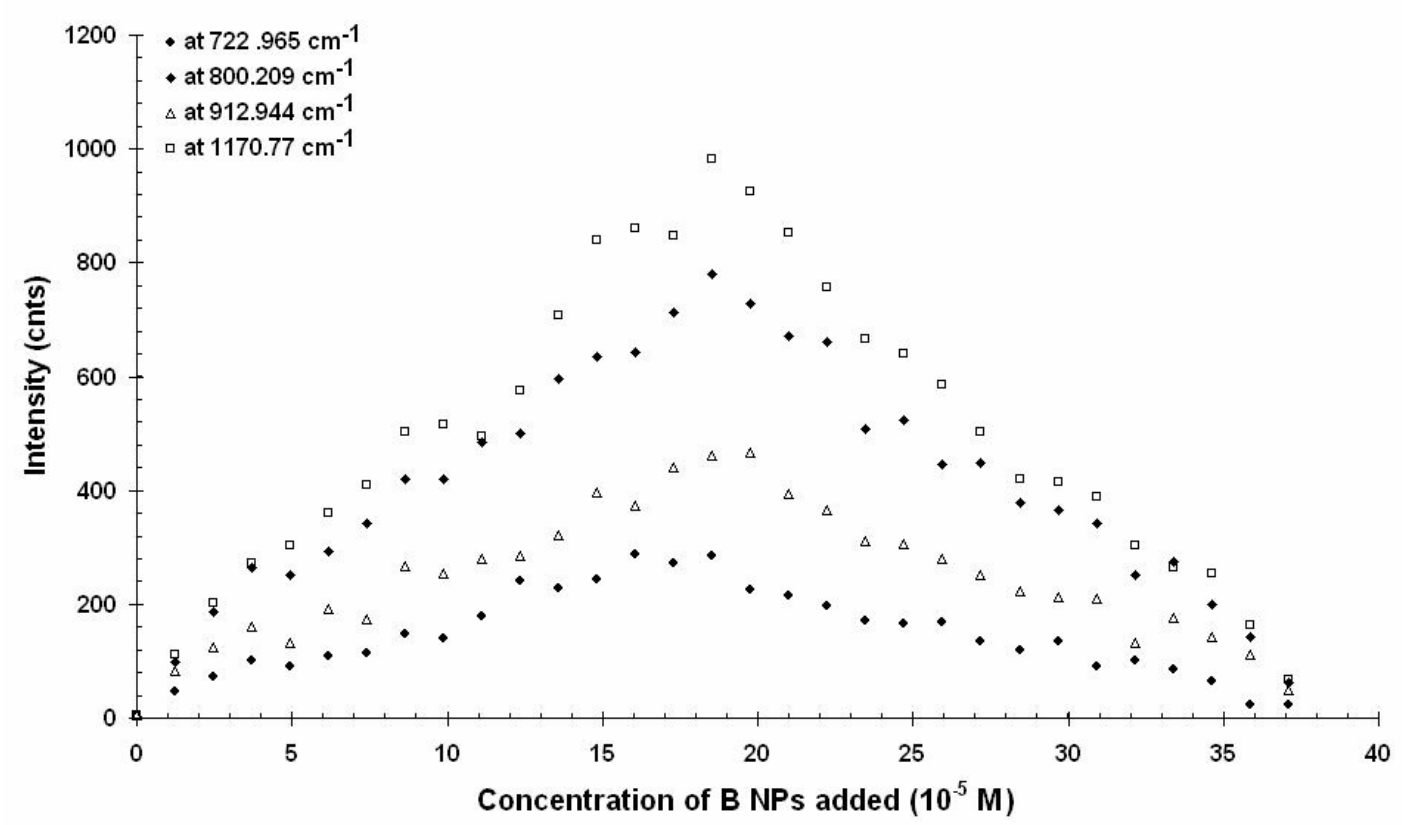

Figure 11. Observed Raman spectra emission trend of $3.33 \times 10^{-5} \mathrm{M}$ CV with increasing concentrations of the blue colloidal nanoparticles with excitation by the $660 \mathrm{~nm}$ laser.

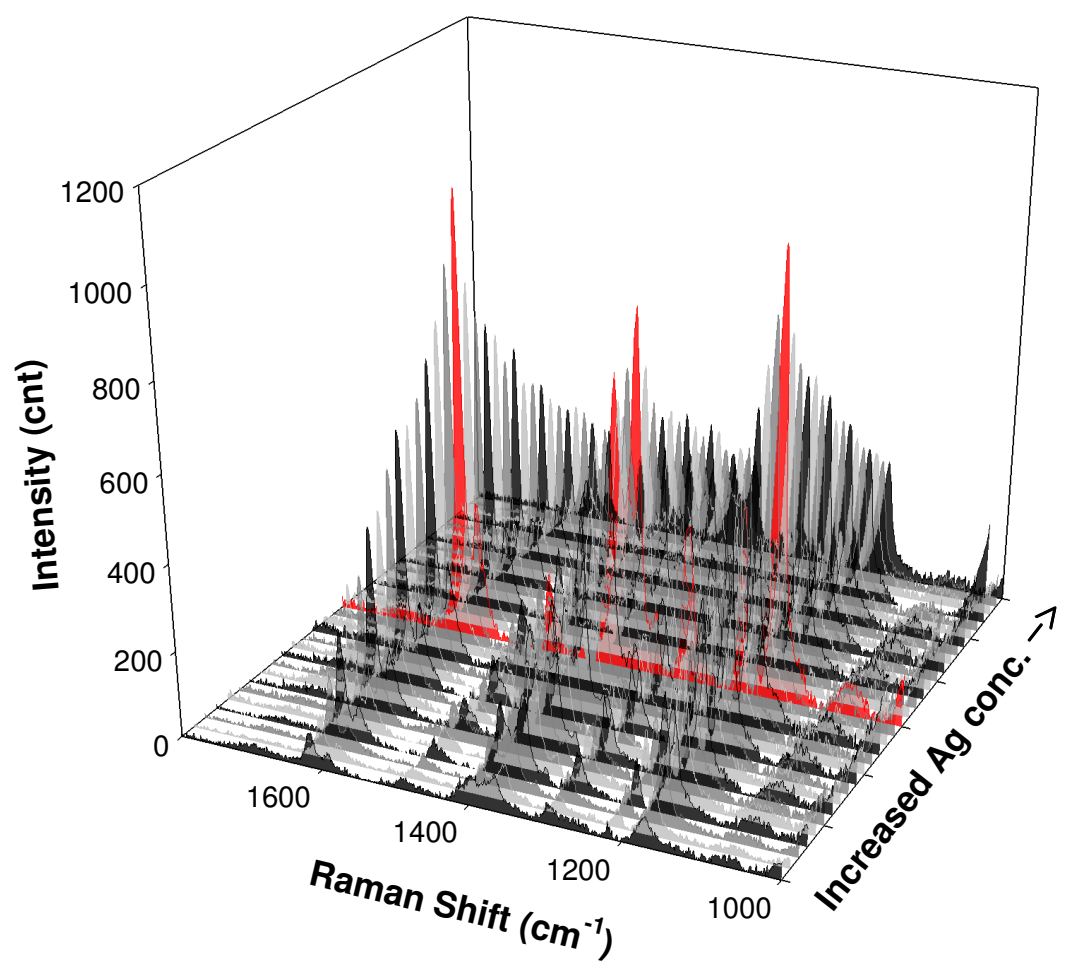

Figure 12. Raman Spectra of $3.33 \times 10^{-5} \mathrm{MMG}$ with increasing concentrations of the blue colloidal nanoparticles with excitation by the $660 \mathrm{~nm}$ laser. The bands of the Raman spectra may be assigned as follows; $\sim 1170 \mathrm{~cm}^{-1}$, in plane aromatic $\mathrm{C}-\mathrm{H}$ bending vibration; $\sim 1365 \mathrm{~cm}^{-1}$, N-C stretch; $\sim 1395 \mathrm{~cm}^{-1}$, $\mathrm{C}-\mathrm{C}$ and $\mathrm{C}-\mathrm{H}$ in plane motion (aromatic); $\sim 1615 \mathrm{~cm}^{-1}, \mathrm{~N}-\mathrm{C}\left(\phi\right.$ bond) and C-C stretch ${ }^{43}$. 


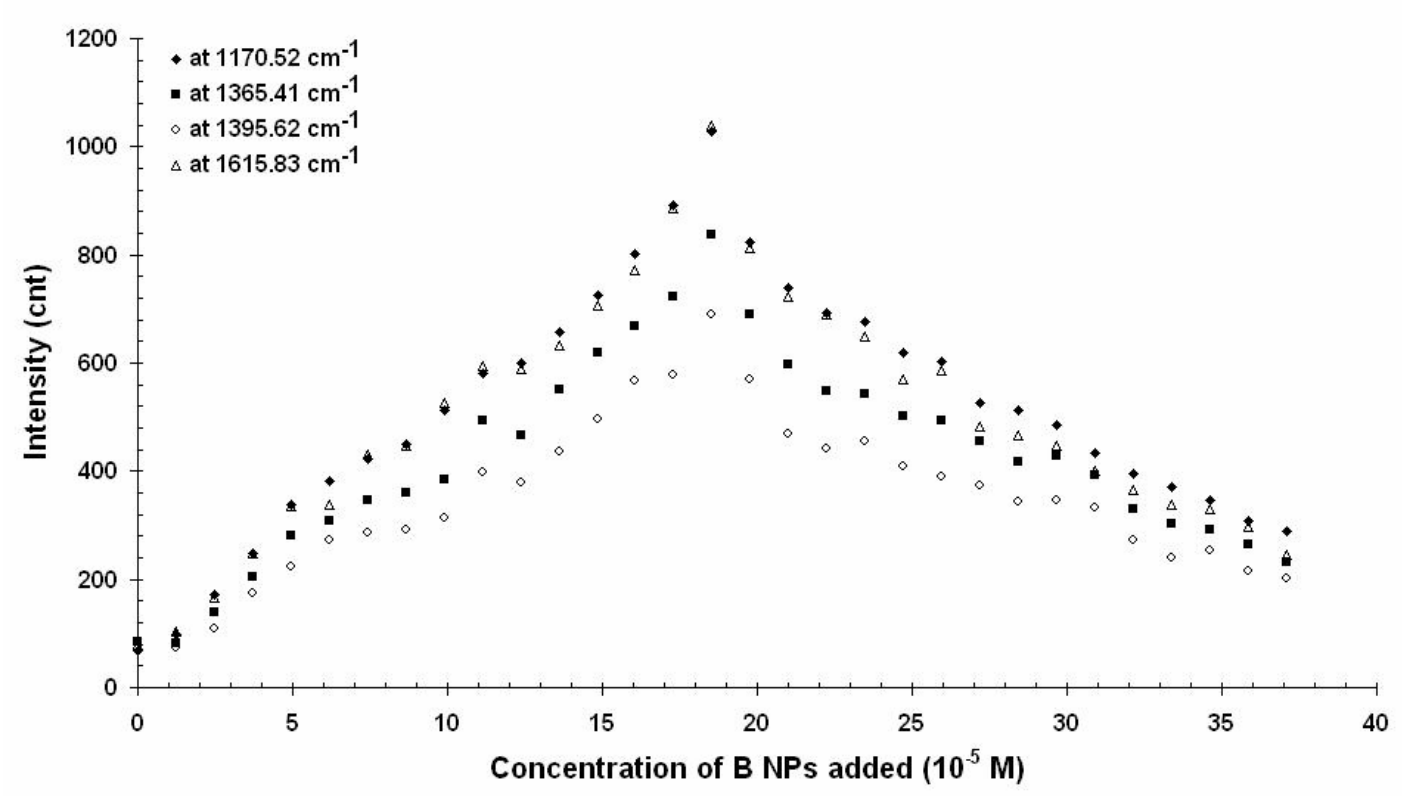

Figure 13. Observed Raman spectra emission trend of $3.33 \times 10^{-5} \mathrm{M}$ MG with increasing concentrations of the colloidal nanoparticles with excitation by the $660 \mathrm{~nm}$ laser.

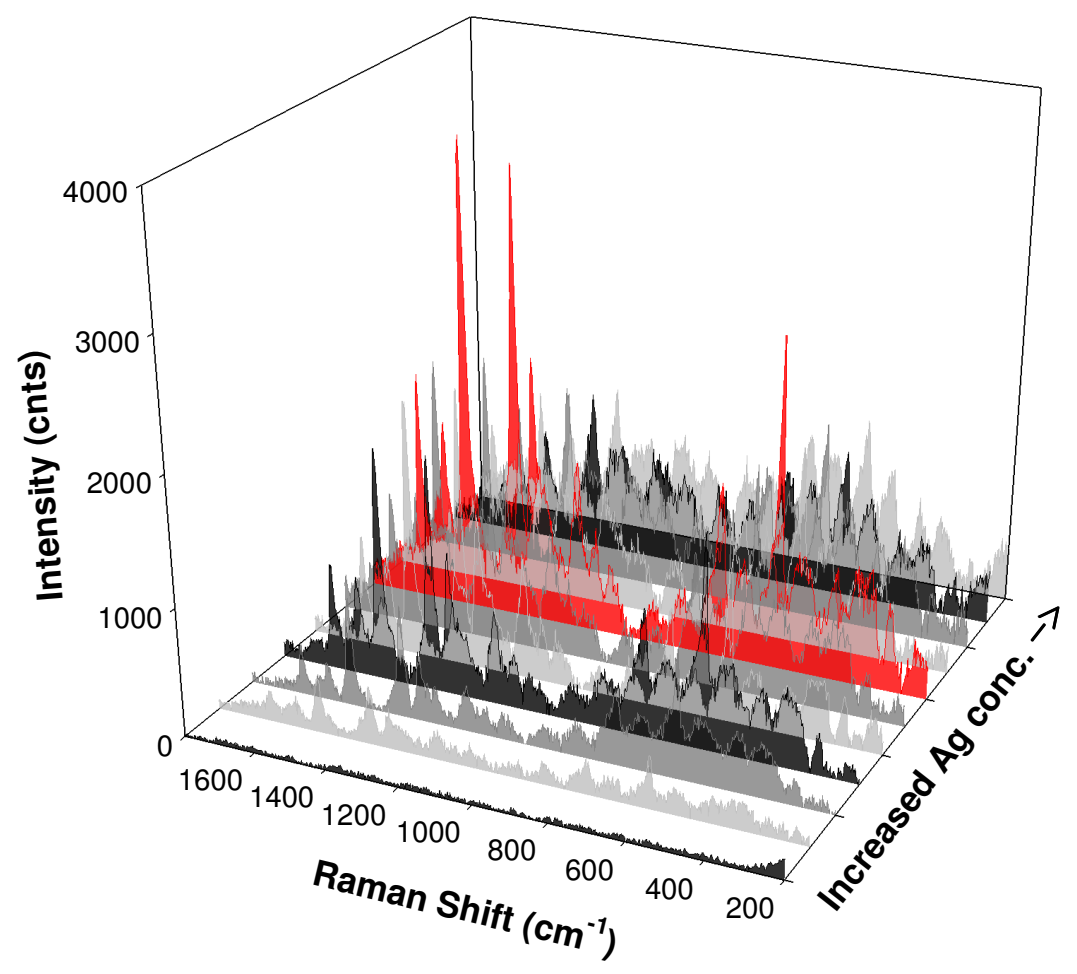

Figure 14. Raman Spectra of $3.33 \times 10^{-5} \mathrm{M}$ R6G with increasing concentrations of the blue colloidal nanoparticles with excitation by the $660 \mathrm{~nm}$ laser. Bands of the Raman spectra may be assigned as follows; $\sim 610 \mathrm{~cm}^{-1}, \mathrm{C}-\mathrm{C}-\mathrm{C}$ in plane aromatic vibration; $\sim 769 \mathrm{~cm}^{-1}, \mathrm{C}-\mathrm{H}$ out of plane bending; $1181 \mathrm{~cm}^{-}$ 
1, C-H in plane bending; $\sim 1316 \mathrm{~cm}^{-1}$ and $\sim 1573 \mathrm{~cm}^{-1}$ in plane bending; $\sim 1361 \mathrm{~cm}^{-1}, \sim 1508 \mathrm{~cm}^{-1}$ and $1648 \mathrm{~cm}^{-1}$, aromatic C-C stretching ${ }^{41}$.

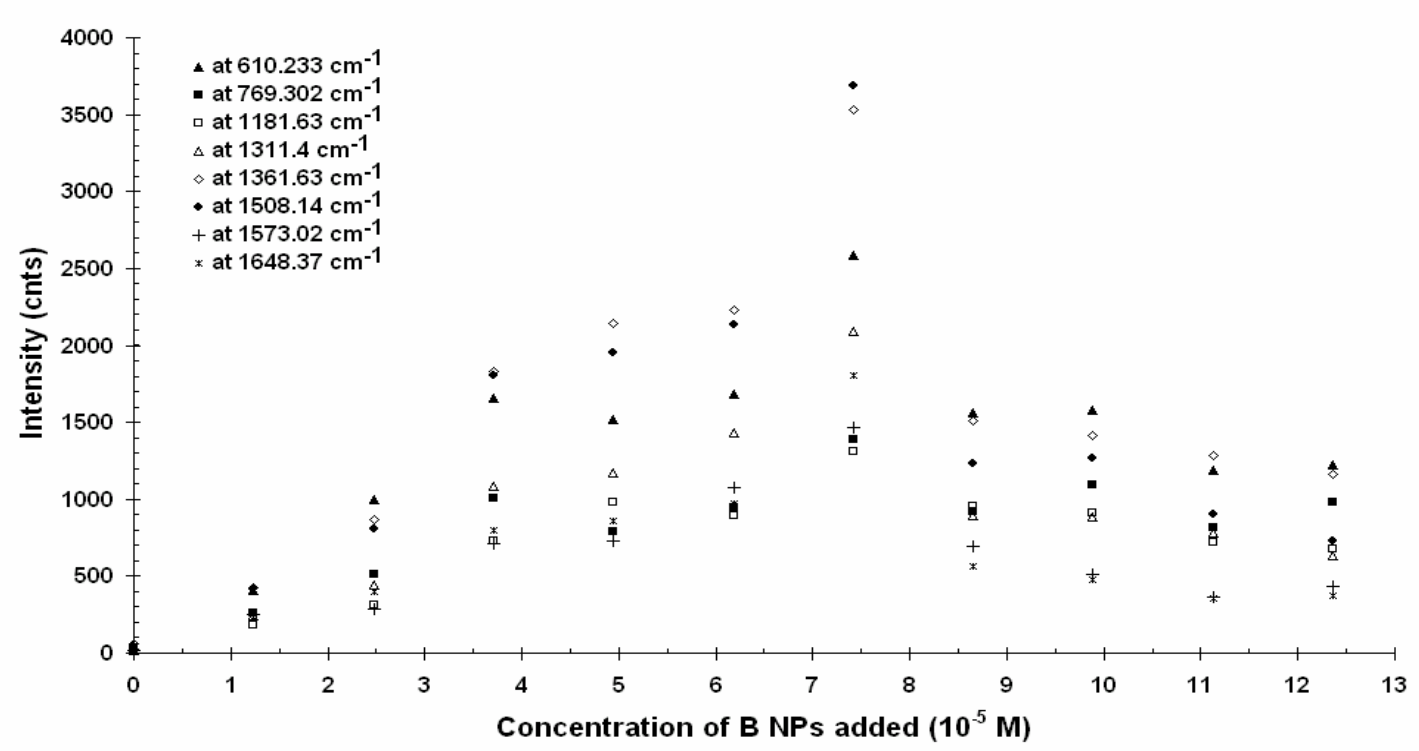

Figure 14. Observed Raman spectra emission trend of $3.33 \times 10^{-5} \mathrm{M}$ R6G with increasing concentrations of the blue colloidal nanoparticles with excitation by the $660 \mathrm{~nm}$ laser.

This is one of the first studies of the effect of changing nanoparticle concentrations while keeping the analyte concentration constant. In all cases, a clear build up of signal intensity is observed until an optimum ratio is achieved, followed by a decline in signal intensity as the concentration of nanoparticles is further increased as seen in figures 10, 12 and 14.

The initial rise in signal can be attributed to the increase in the number of sites onto which the analyte may absorb as the concentration of nanoparticles; following this the observed signal decreases. Several explanations for this observed response are possible, for example an orientation change; at higher numbers of analyte molecules relative to the Silver nanoparticles, the signal is low because of the random orientation of the analyte molecules in terms of the SERRS substrate (silver nanoparticles). That is at low concentrations, the analyte molecules could lie flat on the silver surface. However as the concentration increases, it's possible that the analyte 'stands-up' so although it is still adsorbed to the substrates surface it does so in a different way. Therefore the analyte is less able to benefit from the effect of the plasmons on the polarisation $^{42}$.

This seems unlikely however as a dropping off of signal is observed at higher concentrations of silver, since no change in the spectra was observed (whereas a 
'levelling' of signal would be the expected result, correlating with better known adsorption models like Langmuir).

An alternative explanation could be due to the nature of the nanoparticles themselves. Because coinage metals support plasmons, the interactions of nanoparticles within the colloid system can generate intense electric fields when illuminated producing high SERRS enhancement 22, 42 . Therefore, the continued addition of nanoparticles increases the interaction of nanoparticles with each other resulting in a large 'background' of plasmonic activity, which could mask or shield the very analyte signal that the SERRS technique aims to enhance.

It also must be noted that in each case the maximum SERRS effect is seen for bands associated with the aromatic activity of each dye, bands $\sim 800 \mathrm{~cm}^{-1}$ and $\sim 1170 \mathrm{~cm}^{-1}$ for $\mathrm{CV}$ (figures $9 \& 10$ ), bands $\sim 1170 \mathrm{~cm}^{-1}$ and $\sim 1615 \mathrm{~cm}^{-1}$ for $\mathrm{MG}$ (figures $11 \& 12$ ) and bands $\sim 1361 \mathrm{~cm}^{-1}$ and $\sim 1508 \mathrm{~cm}^{-1}$ for R6G ${ }^{38-40}$ (figures $13 \& 14$ ), implying that the structure of the analyte too has a role in the sensing mechanism. Thus it is unsurprising that $\mathrm{CV}$ and $\mathrm{MG}$ show similar sensing trends as structurally they share common attributes, in contrast R6G is markedly different in structure, (figure 6), from the other model dyes. If we focus on the bands, which undergo the maximum SERRS enhancement as stated above, it is plausible to base the nanoparticle analyte ratio on the number of aromatic components of each dye. The role of the analytes structure is also emphasised when looking at figures 9 and 11, where the dyes CV and MG both show a maximum signal at $\sim 18.5 \times 10^{-5} \mathrm{M} \mathrm{Ag}$ nanoparticles, a maximum signal enhancement at $1170 \mathrm{~cm}^{-1}$ and a maximum enhancement with the $\mathrm{Ag}$ : analyte molar ratio of $\sim 5.56: 1$. Whereas R6G (figure 14) shows a maximum signal at $\sim 7.5 \times 10^{-5}$ M Ag nanoparticles, a maximum signal enhancement at $1508 \mathrm{~cm}^{-1}$ and a maximum enhancement with the $\mathrm{Ag}:$ analyte molar ratio of $\sim 2.25: 1$. The $\mathrm{Ag}:$ dye ratio for R6G is approximately $40 \%$ of that of the other two dyes (CV and MG) which may indicate a different mode of adsorption, and further illustrates the effect of the analytes structure.

\section{Conclusions}

A simple heterogeneous nucleation (building block) process was used to produce silver colloids with tuneable $\lambda$ max's (where only the concentration of one reagent 
$\mathrm{AgNO}_{3}$, is changed). The synthesis was both rapid and repeatable at room temperature with the resulting colloids displaying good stability over time.

The colloids were shown to be useful in the SERRS technique, as synthesised, without the addition of aggregation agents. Significant enhancement of the Raman signal for each dye occurred even with a relatively short sampling time. For each model analyte a clear trend was observed where with increasing concentration of colloid, there resulted an enhancement of signal until an optimum ratio of analyte to colloid was established. This optimum ratio was found to be consistent for each analyte over a range of concentrations and so can be used to further improve sampling times.

As Raman is non destructive and highly selective (providing a spectral fingerprint of the analyte), this enhancement, coupled with the short sampling time clearly demonstrates the usefulness of SERRS, as a rapid and highly sensitive sensing technique, within analytical chemistry.

\section{Acknowledgements}

A.C. Power thanks the ABBEST PhD Scholarship Programme of the Dublin Institute of Technology, and everybody in the FOCAS Institute/DIT, particularly the members of the Applied Electrochemistry Group for their assistance during the experimental work.

\section{References}

1. C. Drake, S. Deshpande, D. Bera, S. Seal, International Materials Reviews, 2007, 52, 289-317.

2. J.H. Lee, Sensors \& Actuators, B, 2009, 140, 319-336.

3. R. Sanci, M. Volkan, Sensors \& Actuators, B, 139, 2009, 150-155.

4. A. Champion, P. Kambhampati, Chemical Society Reviews, 27, 1998, 241 - 250.

5. D.L Stokes, T. Vo-Dinh, Sensors \& Actuators, B, 69, 2000, 28 - 36.

6. C.J. Addison, A.G. Brolo, Langmuir, 22, 2006, 8696 - 8702.

7. D.I. Ellis, R. Goodacre, Analyst, 131, 2006, 875 - 885.

8. S. Feng, R. Chen, J. Lin, J. Pan, G. Chen, Y. Li, M. Cheng, Z. Huang, J. Chen, H. Zeng, Bisensors and Bioelectronics, 25, 2010, 2414 - 2419.

9. A. Kudelski, Talanta, 76, 2008, 1-8 
10. A. Shkilnyy. M. Souce. P. Dubois, F. Warmont, M.L. Saboungi, I. Chourpa, Analyst, 134, 2009, 1868 - 1872.

11. M.E. Abdelsalam, S. Mahajan, P.N. Bartlett, J.J. Baumberg, A.E. Russell, Journal of American Chemistry Society, 129, 2007, 7399 - 7406

12. D.G. Yu, W.C. Lin, C.H. Lin, L.M. Chang, M.C. Yang, Materials Chemistry and Physics, 101, 2007, $93-98$.

13. C.E. Talley, J.B. Jackson, C. Oubre, N.K Grady, C.W. Hollars, S.M. Lane, T.R. Huser, P. Nordlander, N.J. Halas, Nano Letters, 5, 2005, 1569.

14. L.L. Bao, S.M. Mahurin, C.D. Liang, S. Dai, Journal of Raman Spectroscopy, 34, 2003, 394.

15. S. Kundu, M. Mandal, S.K. Ghosh, T. Pal, Journal of Colloid and Interface Science, 272, 2004, 134.

16. A.D. McFarland, M.A. Young, J.A. Dieringer, R.P. Van Duyne, Journal of Physical Chemistry B, 109, 2005, 11279 - 11285.

17. W.E. Smith, Chemical Society Reviews, 37, 2008, 955 - 964.

18. W.C. Lin, M.C. Yang, Macromolecular Rapid Communications, 26, 2005, 1942 1974.

19. P. Hildebrandt, M. Stockburger, Journal of Physical Chemistry, 88, 1984, 5935.

20. A. Otto, A. Bruckbauer, Y.X. Chen, Journal of Molecular Structure, 661, 2003, 501.

21. H.S. Shin, H.J. Yang, S.B. Kim, M.S. Lee, Journal of Colloid and Interface Science, 274, 2004, 89.

22. T. Sugimoto, F. Shib, T. Sekiguchi, H. Itoh, Colloids Surface A, 164, 2000,183.

23. S. Mahajan, R.M. Cole, B.F. Soares, S.H. Pelfrey, A.E. Russell, J.J. Baumberg, P.N. Bartlett, Journal of Physical Chemistry C, 113, 2009, 9284 - 9289.

24. D. Aherne, D.M. Ledwith, M.Gara, J.M. Kelly, Advanced Functional Materials, 18, 2008, 2005-2016.

25. D.M. Ledwith, A.M. Whelan, J.M. Kelly, Journal of Materials Chemistry, 17, 2007, 2459-2464.

26. P.K. Khanna, N. Singh, S. Charan, V.V.V.S. Subbarao, R. Gokhale, U.P. Mulik, Materials Chemistry and Physics, 2005, 93, 117-121.

27. T. Li, H.G. Park, S. Choi, Materials Chemistry and Physics, 2007, 105, 325-330.

28. K.L. Kelly, E. Coronado, L.L. Zhao, G.C. Schatz, Journal of Physical Chemistry $B, 2003,107,668-677$. 
29. Z.H. Mbhele, M.G. Salemane, C.G.C.E. van Sittert, J.M. Nedeljkovic, V.

Djokovic, A.S. Luyt, Chemistry of Materials, 2003, 15, 5019-5024.

30. C.F. Bohren, D.R. Huffman, Absorption and scattering of light by small particles, New York; Chichester: Wiley, (1983).

31. Y. Kunieda, K. Nagashima, N. Hasegawa, Y. Ochi, Spectrochimica Acta Part B, 2009, 64, 744-746.

32. E. Filippo, A. Serra, D. Manno, Sensors \& Actuators, B, 2009, 138, 625-630.

33. Q.F . Zhou, J.C. Bao, J. Xu, Journal of Materials Chemistry, 2002, 12, 384-387.

34. Y.J. Yang, Materials Science and Engineering: B, 2006, 131, 200-202.

35. V.K. Sharma, R.A.. Yngard, Y. Lin, Advances in Colloid and Interface Science, 2009, 145, 83-96.

36. Z.H. Mbhele, M.G. Salemane, C.G.C.E. van Sittert, J.M. Nedeljkovic, V.

Djokovic, A.S. Luyt, Journal of Chemistry Materials, 2003, 15, 5019-5024.

37. P. Atkins, J. de Paula, Atkin's Physical Chemistry, 7th ed., Oxford University Press, Oxford; New York, (2002), 845.

38. http://www.zeiss.com/c12567be0045acf1/ContentsFrame/cbe917247da02a1 cc1256e0000491172 (last accessed December 2010)

39. H.B. Lueck, D.C. Daniel, J.L. McHale, Journal of Raman Spectroscopy, 24, 1993, 363-370

40. M.M. Islam, K. Ueno, H. Misawa, Analytical Sciences, 26, 2010, 19-24.

41. X.Z. Sun, L.H. Lin, Z.C. Li, Z.J. Zhang, J.Y. Feng, Applied Surface Science, 256, 2009, 916-920.

42. W. Plieth, Electrochemistry for Materials Sciences, Elsevier, Amsterdam, (2008), $223-228$.

43. J.J Baumberg, T. Kelf, Y. Sugawara, S. Cintra, M. Abdelsalam, P.N. Bartlett, A.E. Russell, Nano Letters, 5, 2005, 2262 - 2267. 